\title{
Gastrointestinal Stromal Tumors (GISTs) and Extragastrointestinal Stromal Tumors (E-GISTs) - A Review
}

\section{Jagtap Sunil Vitthalrao*}

Professor, Department of Pathology, Krishna Institute of Medical Sciences Karad, Deemed University, Karad, India

*Corresponding author: Dr. Sunil Vitthalrao Jagtap, Krishna Institute of Medical Sciences, Deemed University, karad-415110. Maharashtra, India, Tel: 91 - 9960628672; Email: drsvjagtap@gmail.com

\section{Review Article \\ Volume 5 Issue 1}

Received Date: October 13, 2020

Published Date: February 04, 2021

DOI: $10.23880 /$ cprj-16000128

\section{Abstract}

GISTs (Gastrointestinal Stromal Tumors) are the most frequently encountered mesenchymal tumor of the gastrointestinal tract. Gastrointestinal stromal tumors have been recognized as a biologically distinctive tumor type, different from smooth muscle and neural tumors of the gastrointestinal tract (GIT). GISTs present $0.1 \%-3.0 \%$ of gastrointestinal malignant tumors. The goals in treating patients with GIST are to maximize the chance of cure, minimize recurrence. A multidisciplinary approach to patients with GISTs is necessary to optimize the timing of medical and surgical therapy as they have unpredictable behavior. Also long term follow up is essential for all patients, independent of their benign or malignant characteristics.

This review highlight on clinical, diagnostic imaging, histopatholpgical, immunohistochemical ,new markers and management modalities for Gastrointestinal Stromal Tumors (GISTs) and Extragastrointestinal stromal tumors (E-GISTs).

Keywords: Gastrointestinal stromal tumors; extra-GISTs; c-KIT mutations; Mesenchymal tumors

Abbreviations: GISTs: Gastrointestinal stromal tumors; EGISTs: Extra Gastrointestinal stromal tumors; SDH: Succinate dehydrogenase-resistant; TKI: Tyrosine kinase inhibitor; ICC: Interstitial cells of Cajal

\section{Introduction}

Gastrointestinal stromal tumors (GISTs) originate from interstitial cells of Cajal (ICC), which are pacemaker cells that control gastrointestinal track (GIT) peristalsis. GIST occurs throughout the GI tract, from the esophagus to the anus, whereas Extra Gastrointestinal stromal tumors (EGISTs) are tumour without any connection with the intestinal wall.

Miettinen, et al. first defined soft tissue tumors, which originate outside of the GIT and present clinic pathological features and molecular characteristics similar to those of GISTs, as EGISTs [1]. GISTs are defined as morphologically spindle cell, epithelioid or occasionally pleomorphic mesenchymal tumors originated from the intestinal cells of Cajal/related stem cells that usually express C- Kit (CD - 177) protein in $95 \%$ of the cases regardless of the site of origin, histological appearance and biological behavior. Kindblom and associates in 1998 described the interstitial cells of Cajal are mesenchymal tumors of the gastrointestinal tract and have found to have $c$-KIT mutations and KIT (CD117) expression [2]. Recently PDGFRA mutations, SDH alterations, and other drive mutations were identified. Various protein markers such as DOG1, PKC $\theta$ were found to be expressed in GISTs which might help clinicians diagnose CD117negative GISTs [3]. 


\section{Clinical Pathology \& Research Journal}

\section{Review}

Gastrointestinal stromal tumors (GISTs) are uncommon are a heterogeneous group of tumors originate from interstitial cells of Cajal (GI pacemaker) or more primitive stem cells from which both Cajal cells and smooth muscle cells . GISTs accounts for $0.1 \%$ to $3 \%$ of all gastrointestinal neoplasm. These tumors are composed of either spindled or epithelioid cells that have distinctive histologic appearances and clinical behaviours in different portions of the gastrointestinal tract.

On location of tumors GISTs are of either Gastrointestinal or Extragastrointestinal. The GIST are predominantly found in stomach $(60-70 \%)$ followed by ileum and jejunum (25$30 \%)$, colon and rectum (5-15\%), duodenum (5\%) and esophagus (2\%) [4]. The stromal tumors arising outside the gastrointestinal tract are extremely rare .GISTs rarely (about $5 \%$ ) arises within the abdominal cavity without an apparent connection to the GI tract, these are known as Extra-GISTs (EGISTs). For these the metastasis should be carefully ruled out. The various sites for EGISTs are mesentery, omentum, peritoneum, gall bladder, liver, pancreas, spleen, mesocolon, mediastinum and in the pelvis [5-7]. Approximately 80\% of EGISTs are located in the omentum or mesentery. The multifocal presentation of the malignant EGISTs has been reported. The E-GISTs are cytologically and histologically similar to GISTs but usually behave more aggressively, with high metastatic potential and a high recurrence rate.

Clinically about 35\% are detected incidentally, while large tumour present as vague abdominal discomfort or pain (20-50\%), acute or chronic GI bleeding, intestinal obstruction $(20 \%)$ or altered bowel habits, and rarely perforation with peritonitis [8-10]. Spontaneous rupture is very rare manifestation of a GIST. We had a case of malignant GIST of sigmoid colon with perforation and peritonitis. EGISTs lack mucosal involvement and are therefore often asymptomatic as compared to GISTs.

The occurrence of GISTs ranging from small benign nodules to malignant tumors in all sites. Histopathologically characteristics pattern is spindle cell type (70\%) appearing as fusiform cells in intersecting whorls. While other patterns seen are epithelioid type (20\%) appearing as rounded cells in a nested pattern ,mixed pattern are noted in $10 \%$ of cases [11]. Associated changes of myxoid, chondroid, hyalinized blood vessel walls, calcification, necrosis is frequently noted. Histopathological morphology, immune phenotype and molecular characteristics of EGIST are similar to GISTs.

On histomorphological features differential diagnosis of GIST include desmoid fibromatosis, leiomyoma/sarcoma, schwannoma, intra-abdominal fibromatosis, paragangliomas, inflammatory myofibroblastic tumor, inflammatory fibroid polyp, myxofibrosarcoma, solitary fibrous tumor, synovial sarcoma $[12,13]$.

GISTs on imaging techniques -abdominal ultrasound, CT enterography, magnetic resonance imaging, and positron emission transverse tomography are identify for its site, invasion, tumor metastasis or complication like perforation.

The most important cut-offs as indicators of aggressive clinical behavior were tumor size of $5 \mathrm{~cm}$ and 5 mitoses/50 HPF. The Asian consensus guideline indicated that all GISTs may have malignant potential [14]. Recently new group of GIST was added is "SDH -succinate dehydrogenase-resistant GIST".

In general the diagnosis is based on characteristic histological findings and expression of transmembrane receptor tyrosine kinase protein (CD 117) derived from activated c-kit gene. It makes the KIT to be a very specific and sensitive marker in the differentiating GIST from other mesenchyma tumors in the GI tract. The IHC plays a key role in the final diagnosis of GIST. Immunohistochemically GISTs uniformly express CD117 (95\%), CD 34(70\%) and SMA (30$40 \%$ ).The $5 \%$ of tumors are positive for PDGFR mutation [15]. These are key to effective therapeutic intervention of GISTs. New markers DOG1 and PKC-theta are expressed in KIT negative cases [16].

The entity micro-GISTs (tumor $<1 \mathrm{~cm}$ ) have low or no mitotic activity and have little clinical significance. Cytogenetically mutation of KIT or PDGFRA occurs in micro GIST (1-10mm [17].

Currently tumor less than or equal to $2 \mathrm{~cm}$ or with less than 5 mitosis per $50 \mathrm{HPF}$ are thought to have lower malignant and metastatic potential. GIST with larger size ( $>10 \mathrm{~cm}$ in diameter) or with mitotic count $>10 / 50 \mathrm{HPF}$ and GIST with diameter $>5 \mathrm{~cm}$ and 5 mitotic figure/ $50 \mathrm{HPF}$ are considered at high risk for recurrence as modified Armed Forces Institute of Pathology risk criteria [18].

The tumor size, mitotic count and site determine the risk assessment, prognostic factor and when adjuvant drug therapy is required. The revised NIH criteria was based on Joensuu's proposal for modification based on nongastric tumors $(2.1-5 \mathrm{~cm}+>5$ mitoses per $50 \mathrm{HPF}$ or $5.1-10 \mathrm{~cm}+$ $\leq 5 / 50 \mathrm{HPF}$ ) and tumor rupture have high-risk category [19].

The gold standard treatment of GISTs is surgical resection. Specific therapy targeting the kit receptor with Imatinib has resulted in improved prognosis for patients with unresectable, metastatic and recurrent disease [20]. GIST presented with perforation and peritonitis is 


\section{Clinical Pathology \& Research Journal}

extremely rare phenomenon. Tyrosine kinase inhibitor (TKI) therapy benefits the substantial improvements in survival, both for patients with localized GIST and those with advanced disease. Advanced GIST but have no or limited efficacy in patients with the PDGFRA D842V mutation or patients with GIST lacking KIT and PDGFRA mutations [21]. EGIST patients present at early age, relatively larger tumors and a poorer prognosis as compared with GIST.

The accurately molecular diagnosis of GISTs guides clinicians to precision medicine and provides optimal treatment for the patients with GISTs. Imatinib mesylate, sunitinib and regorafenib are selective tyrosine kinase receptor inhibitor (TKI), are used as an adjuvant or neoadjuvant therapy. These improved the morbidity and mortality associated with GISTs. Ponatinib has activity against BCR-ABL, CD117 exon 11 mutations and PDGFRA. The favorable prognosis of a GIST is associated with early histological diagnosis and adequate treatment.

This review highlight on clinical, diagnostic imaging, histopatholpgical, immunohistochemical, new markers and management modalities.

\section{References}

1. Miettinen M, Lasota J (2006) Gastrointestinal stromal tumors: pathology and prognosis at different sites. Semin Diagn Pathol 23(2): 70-83.

2. Kindblom LG, Remotti HE, Aldenborg F, Meis-Kindblom JM (1998) Gastrointestinal pacemaker cell tumor (GIPACT): gastrointestinal stromal tumors show phenotypic characteristics of the interstitial cells of Cajal. Am J Pathol 152(5): 1259-1269.

3. Wu CE, Tzen CY, Wang SY, Yeh CN (2019) Clinical Diagnosis of Gastrointestinal Stromal Tumor (GIST): From the Molecular Genetic Point of View. Cancers (Basel) 11(5): 679.

4. Miettinen M, Lasota J (2001) Gastrointestinal Stromal Tumor-Definition, Clinical, Histological, Immunohistochemical, and Molecular Genetic Features and Differential Diagnosis. Virchows Arch 438(1): 1-12.

5. Beham AW, Schaefer IM, Schüler P, Cameron S, Ghadimi BM (2012) Gastrointestinal stromal tumors. Int J Colorectal Dis 27(6): 689-700.

6. DeMatteo RP, Lewis JJ, Leung D, Mudan SS, Woodruff JM, et al. (2000) Two hundred gastrointestinal stromal tumors: recurrence patterns and prognostic factors for survival. Ann Surg 231(1): 51-58.

7. Barros A, Linhares E, Valadão M, Gonçalves R, Vilhena
B, et al. (2011) Extragastrointestina stromal tumors (EGIST): a series of case reports. Hepatogastroenterology 58(107-108): 865-868.

8. Jagtap SV, Nikumbh DB, Kshirsagar AY, Bohra A, Khatib W (2012) Malignant Gastrointestinal Stromal tumor of the Sigmoid Colon with Perforation and Peritonitis-An Unusual Presentation. International Jr of Health Sciences and Research 2(3): 104-109.

9. Ricci R (2016) Syndromic gastrointestinal stromal tumors. Hered Cancer Clin Pract 14: 15.

10. Laperouse P, Raines D, Diamond K, Rivera S, Newman W, et al. (2008) Gastrointestinal Stromal Tumors: A Case Report and Review of the Literature. J La State Med Soc 160(3): 128-133.

11. Fletcher CD, Berman JJ, Corless C, Gorstein F, Lasota J, et al. (2002) Diagnosis of gastrointestinal stromal tumors: a consensus approach. Hum Pathol 33(5): 459-465.

12. Jagtap SV, Jain A, Jagtap SS, Kshirsagar AY (2015) Highgrade myxofibrosarcoma-presented as a large mass of right upper arm. Indian J Pathol Microbiol 58(1): 105107.

13. Turner MS, Goldsmith JD (2009) Best practices in diagnostic immunohistochemistry: spindle cell neoplasms of the gastrointestinal tract. Arch Pathol Lab Med 133(9): 1370-1374.

14. Nishida $T$ (2018) Asian consensus guidelines for gastrointestinal stromal tumor: what is the same and what is different from global guidelines. Transl Gastroenterol Hepatol 3: 11.

15. Heinrich MC, Corless CL, Duensing A, McGreevey L, Joseph N, et al. ( 2003) PDGFRA activating mutations in gastrointestinal stromal tumors. Science 299(5607): 708-710.

16. Espinosa I, Lee CH, Kim MK, Rouse BT, Subramanian S, et al. (2008) A novel monoclonal antibody against DOG1 is a sensitive and specific marker for gastrointestinal stromal tumors. Am J Surg Pathol 32(2): 210-218.

17. Liegl B, Hornick JL, Corless CL (2009) Monoclonal antibody DOG1.1 shows higher sensitivity than KIT in the diagnosis of gastrointestinal stromal tumors, including unusual subtypes. Am J Surg Pathol 33(3): 437-446.

18. Goh BK, Chow PK, Yap WM, Kesavan SM, Song IC, et al. (2008) Which is the optimal risk stratification system for surgically treated localized primary GIST? Comparison of three contemporary prognostic criteria in 171 tumors and a proposal for a modified Armed Forces Institute of Pathology risk criteria. Ann Surg 
Oncol 15(8): 2153-2163.

19. Joensuu H (2008) Risk stratification of patients diagnosed with gastrointestinal stromal tumor. Hum Pathol 39(10): 1411-1419.

20. Lanke G, Lee JH (2017) How best to manage gastrointestinal stromal tumor. World J Clin Oncol 8(2):
135-144.

21. Huss S, Pasternack H, Ihle MA, Merkelbach-Bruse S, Heitkötter B, et al. (2017) Clinicopathological and molecular features of a large cohort of gastrointestinal stromal tumors (GISTs) and review of the literature: BRAF mutations in KIT/PDGFRA wild-type GISTs are rare events. Hum Pathol 62: 206-214. 